\section{Commentary: Taking matters into your own hands: Venoarterial extracorporeal membrane oxygenation for controlled reperfusion during lung transplantation}

\author{
Brian Mitzman, MD
}

Primary graft dysfunction (PGD) remains a dreaded complication after lung transplantation. In this issue of the Journal, Hoetzenecker and colleagues describe using venoarterial (VA) extracorporeal membrane oxygenation (ECMO) support for bilateral lung transplantation at a single major European center. ${ }^{1}$ The main goal was to evaluate primary graft dysfunction and whether the use of routine ECMO with controlled reperfusion of the grafts would improve midterm outcomes. The authors prospectively evaluated 159 patients over approximately 19 months. This is an impressive number of transplantations in a short period, rivalling the highestvolume centers in the United States.

All recipients underwent central cannulation before implantation. Flow was initially set to $40 \%$ of cardiac output and was reduced to $1 \mathrm{~L}$ of flow after implantation of the first graft. During the second implantation, flow was adjusted to keep pulmonary systolic pressures below $25 \mathrm{~mm} \mathrm{Hg}$. ECMO was temporarily discontinued after the graft completion. If any signs of dysfunction occurred, cannulas were transferred to the femoral vein and artery for postoperative support. The authors calculated PGD at 4 different time points, with an impressively low rate of graft dysfunction seen. By 72 hours, $76.7 \%$ were extubated, only 2 patients were PGD3, and only 1 of 13 remained on extended ECMO. The authors conclude that ECMO is a safe adjunct, with only 6 ECMO-related complications

From the Department of Cardiothoracic Surgery, NYU Langone Health, New York, NY

Disclosures: Author has nothing to disclose with regard to commercial support.

Received for publication Nov 6, 2019; revisions received Nov 6, 2019; accepted for publication Nov 7, 2019; available ahead of print Nov 28, 2019.

Address for reprints: Brian Mitzman, MD, Department of Cardiothoracic Surgery, NYU-Winthrop Hospital, 259 1st St, Mineola, NY 11501 (E-mail: Brian. mitzman@nyulangone.org).

J Thorac Cardiovasc Surg 2020;160:330-1

$0022-5223 / \$ 36.00$

Copyright (c) 2019 by The American Association for Thoracic Surgery

https://doi.org/10.1016/j.jtcvs.2019.11.037

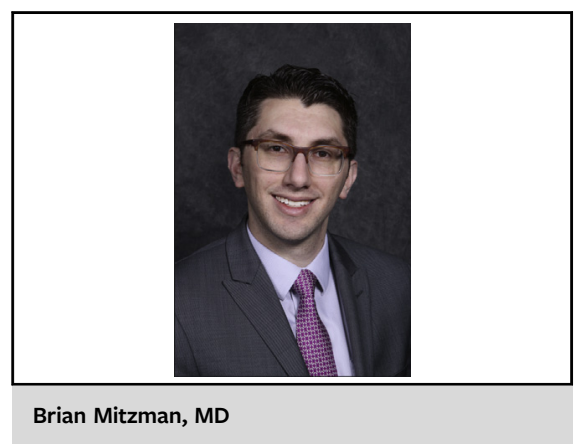

CENTRAL MESSAGE

Many times, less is more. In this

case, using venoarterial extra-

corporeal membrane oxygena-

tion to alter reperfusion can

significantly improve primary

graft dysfunction rates and

overall survival.

(5 groin infections and 1 aortic injury). There were no thromboembolic events (despite minimal heparin dosing and no checks of ACT) or bleeding requiring intervention. Two-year survival was calculated as $86 \%$.

The authors first published a retrospective review of 6 years' of lung transplantation at their center in $2018 .{ }^{2}$ A total of 582 bilateral lung transplant recipients were evaluated in three subgroups: no ECMO, intraoperative ECMO, and interoperative plus prolonged postoperative ECMO. The intraoperative ECMO group showed improved survival at all time points, with the greatest increase seen at 1 year $(91 \%$ vs $82 \%)$. These superb results prompted the change in protocol to the use of ECMO for all bilateral transplantations. It also led to ethical reservations related to the use of a comparative arm in the current prospective study.

Can some lung transplantations be performed without any additional intraoperative support? Of course they can. ECMO as an adjunct to transplantation for technical ease of graft insertion is not the point of this analysis. What the authors show is that the use of intraoperative ECMO led to increased survival and decreased PGD, with minimal ECMO-related complications. This has been confirmed in several other studies. ${ }^{3-6}$ The exact reason for the improved outcomes is unknown. Whether it is truly the controlled reperfusion with VA ECMO or just increased oxygenation (which can be achieved with venovenous ECMO) remains to be evaluated. 
Although this study does not provide sufficient evidence to make intraoperative ECMO the gold standard, it certainly adds credence to the view that this significant additional intervention can improve outcomes with little risk. I applaud the authors for their high- volume excellent outcomes, and their addition to this important body of research.

\section{References}

1. Hoetzenecker K, Benazzo B, Stork T, Sinn K, Schwarz S, Schweiger T, et al; Vienna Lung Transplant Group. Bilateral lung transplantation on intraoperative extracorporeal membrane oxygenator: an observational study. J Thorac Cardiovasc Surg. 2020;160:320-7.e1.

2. Hoetzenecker K, Schwarz S, Muckenhuber M, Benazzo A, Frommlet M, Schweiger $\mathrm{T}$, et al. Intraoperative extracorporeal membrane oxygenation and the possibility of postoperative prolongation improve survival in bilateral lung transplantation. J Thorac Cardiovasc Surg. 2018;155 2193-306.

3. Nazarnia S, Subramaniam K. Pro: veno-arterial extracorporeal membrane oxygenation (ECMO) should be used routinely for bilateral lung transplantation. J Cardiothorac Vasc Anesth. 2017;31:1505-8.

4. Hashimoto K, Hoetzenecker K, Yeung JC, Jeagal L, Donahoe L, Pierre A, et al Intraoperative extracorporeal support during lung transplantation in patients bridged with venovenous extracorporeal membrane oxygenation. J Heart Lung Transplant. 2018;37:1418-24.

5. Salman J, Ius F, Sommer W, Siemeni T, Kuehn C, Avsar M, et al. Mid-term results of bilateral lung transplant with postoperatively extended intraoperative extracorporeal membrane oxygenation for severe pulmonary hypertension. Eur J Cardiothorac Surg. 2017:52:163-70.

6. Moser B, Jaksch P, Taghavi S, Murakozy G, Lang G, Hager H, et al. Lung transplantation for idiopathic pulmonary arterial hypertension on intraoperative and postoperatively prolonged extracorporeal membrane oxygenation provides optimally controlled reperfusion and excellent outcome. Eur J Cardiothorac Surg. 2018;53:178-85.

\section{Commentary: Central defense} on trial

\author{
Ankit Dhamija, MD, ${ }^{\mathrm{a}}$ \\ Heather K. Hayanga, MD, MPH, ${ }^{\mathrm{b}}$ \\ Kelsey Musgrove, MD, ${ }^{\mathrm{a}}$ and \\ J. W. Awori Hayanga, MD, MPH
}

In their article in this issue of the Journal, Hoetzenecker and colleagues ${ }^{1}$ evaluate the utility of intraoperative extracorporeal membrane oxygenation in lung transplantation, endorsing its routine use as a means of reducing primary graft dysfunction. This analysis is timely and relevant and is presented against a backdrop of high-volume transplantation. Their results, although commendable, remain nonetheless susceptible to critique, particularly from a methodologic standpoint. In the quest to establish a paradigm of care, Hoetzenecker and colleagues ${ }^{1}$ gambled against including neither a comparative nor a control arm.

\footnotetext{
From the ${ }^{a}$ Department of Cardiovascular and Thoracic Surgery, West Virginia University, Morgantown, WVa; and ${ }^{\mathrm{b}}$ Division of Cardiac Anesthesia, WVU Heart and Vascular Institute, West Virginia University, Morgantown, WVa.

Disclosures: Authors have nothing to disclose with regard to commercial support.

Received for publication Sept 27, 2019; revisions received Sept 27, 2019; accepted for publication Sept 29, 2019; available ahead of print Oct 9, 2019.

Address for reprints: J. W. Awori Hayanga, MD, MPH, West Virginia University

School of Medicine, 1 Medical Center Dr, Morgantown, WV 26505-8059

(E-mail: jeremiah.hayanga@wvumedicine.org).

J Thorac Cardiovasc Surg 2020;160:331-2

$0022-5223 / \$ 36.00$

Copyright (c) 2019 by The American Association for Thoracic Surgery

https://doi.org/10.1016/j.jtcvs.2019.09.124
}

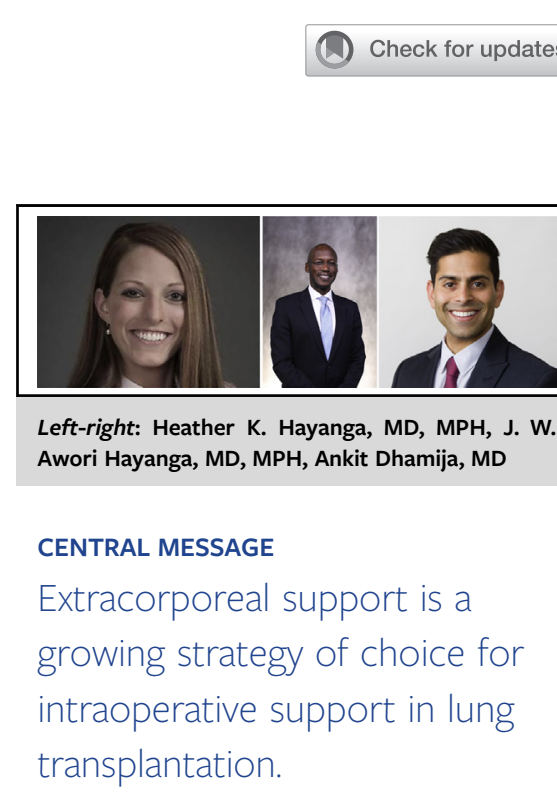

As such, they expose themselves to unmitigated confounding and bias. Their analysis is shielded from the tempering, moderating benefit of randomization, which might also otherwise have afforded a more objective lens through which to view their findings. This is particularly to the point in the weight placed on the comparison with cardiopulmonary bypass. The absence of propensity matching further compounds the confounding exacerbated by the prevailing fleeting consensus regarding the definition of primary graft dysfunction. $^{2}$ 